\title{
Fourier Transform Near-Infrared Absorption Spectroscopic Study of Catalytic Isomerization of Quadricyclane to Norbornadiene by Copper(II) and Tin(II) Salts
}

\author{
Eric Chau-Chin Chuang and King-Chuen Lin* \\ Department of Chemistry, National Taiwan University, and Institute of Atomic and Molecular Sciences, \\ Academia Sinica, Taipei 106, Taiwan, Republic of China
}

Received: June 11, 2001

\begin{abstract}
By using Fourier transform near-infrared (NIR) absorption spectroscopy, we have investigated the catalytic conversion of quadricyclane to norbornadiene. Either $\mathrm{CuSO}_{4}$ in chloroform or $\mathrm{SnCl}_{2}$ in benzene is used as catalyst. To avoid the effect of sample heterogeneity, the reaction mixture is kept still without stirring. The NIR light beam is guided to propagate through the solution right above the surface of metal salt. The NIR absorption spectra are acquired at 5-min intervals for a reaction period of $6 \mathrm{~h}$. The related concentrations of quadricyclane and norbornadiene in the temporal evolution are determined with the method of partial least squares. A kinetic model for the pseudo-first-order reaction is derived considering the diffusion motion. Accordingly, the second-order rate constant for the isomerization catalyzed by $\mathrm{CuSO}_{4}$ and $\mathrm{SnCl}_{2}, \mathrm{respectively}$ are determined to be $(1.38 \pm 0.04) \times 10^{-3}$ and $(4.62 \pm 0.09) \times 10^{-3} \mathrm{~min}^{-1} \mathrm{~g}^{-1}$. The norbornadiene is produced via a one-site coordination between the reactant and the catalyst. The product contribution from the intermediate of a two-site coordination is negligible in our system. The obtained result for $\mathrm{CuSO}_{4}$ is comparable with that detected by using Raman spectroscopy.
\end{abstract}

\section{Introduction}

The interconversion of quadricyclane (Q) and norbornadiene (N) has attracted considerable attention because of its potential for solar energy storage. ${ }^{1,2}$ Energy is stored when norbornadiene is photochemically converted to quadricyclane, ${ }^{3-5}$ which is relatively stable at room temperature with a lifetime of $140 \mathrm{~h}$ at $100{ }^{\circ} \mathrm{C}$. The energy is then released when quadricyclane is catalytically converted back to norbornadiene. ${ }^{6-8}$

In a practical design of a solar energy system based on valence isomerization, the catalyst desired for the conversion of quadricyclane should fit two conditions. ${ }^{1}$ First, the reaction is rapid without byproducts, which may reduce the conversion efficiency and probably precipitate to cause the cleaning problem. Second, the photochemical reaction chamber and the catalytic conversion chamber must be kept apart. Thus, the catalyst should remain insoluble in the reaction medium to avoid contamination of the other photochemical reaction chamber. In this sense, transition metal salts such as $\mathrm{Sn}, \mathrm{Cu}, \mathrm{W}, \mathrm{Mo}, \mathrm{Co}$, $\mathrm{Ni}, \mathrm{Rh}$, and $\mathrm{Pd}$ are found to be potential candidates. ${ }^{1,6-12}$ Among them, the $\mathrm{Sn}$ (II) and $\mathrm{Cu}$ (II) salts are cheap catalysts and frequently used for study. Moore and co-workers reported that the hydrated $\mathrm{Cu}(\mathrm{II})$ and $\mathrm{Sn}(\mathrm{II})$ salts cannot catalyze the isomerization. ${ }^{6}$ A coordination site on the metal must be available for the catalytic conversion. In addition, the copper chloride and bromide may lead to the side products of $\mathrm{C}_{7} \mathrm{H}_{8} \mathrm{X}_{2}$ and $\mathrm{CuX}(\mathrm{X}=\mathrm{Cl}$ and $\mathrm{Br})$, such that the depletion rate of quadricyclane becomes larger than the production rate of norbornadiene. ${ }^{6}$ The catalytic surface also changes to white color. To avoid the above effects, in this work we choose anhydrous tin(II) chloride and copper(II) sulfate to catalyze quadricyclane.

The kinetics of the conversion of quadricyclane to norbornadiene by copper and tin salts has been investigated. ${ }^{6-8,13}$

* Author to whom correspondence should be addressed. Fax: 886-2 236 21483. E-mail: kclin@mail.ch.ntu.edu.tw.
Moore and co-workers have determined the quadricyclane and norbornadiene concentrations by gas chromatography equipped with both flame ionization and mass spectroscopy detection. ${ }^{6}$ Their procedure requires the removal and analysis of the aliquots of the reaction mixture at intervals during the reaction. To offer the potential for automated, nondestructive, continuous monitoring of the isomerization reaction, Vickers and co-workers demonstrated with fiber-optic Raman spectroscopy. ${ }^{7}$ Nevertheless, the suspended solids increased the sample opacity. Such effect of sample heterogeneity did not change much the detection limit, but decreased the measurement sensitivity. ${ }^{7}$

To avoid the effect of sample heterogeneity, in this work we acquire Fourier transform near-infrared (NIR) absorption spectra for the isomerization reaction of quadricyclane without stirring the suspended catalysts. The $\mathrm{CuSO}_{4}$ and $\mathrm{SnCl}_{2}$ catalysts are remaining still in the reaction medium. The reactants diffuse in the solid stacks to undergo catalytic isomerization on the metal surface, while the products are probed outside the solid stacks. The quadricyclane and norbornadiene concentrations in the reaction evolution may be determined with the aid of partial least-squares method. A kinetic model involving the diffusion motion is derived. On the basis of this model, the conversion rate constants for these two catalysts are determined and compared with previous measurements.

\section{Experiment}

A step-scan Fourier transform spectrometer (FTS, IFS-88, Bruker) was implemented with a tungsten lamp as the NIR radiation source and a $\mathrm{Si}$ diode detector available for the wavelength range from 6000 to $25000 \mathrm{~cm}^{-1}$. The FTS contains a classical Michelson interferometer, which can be operated in either rapid-scan or step-scan mode. ${ }^{14-16}$ In this work a rapidscan mode was used. The obtained interferogram in each scan, as completed within milliseconds, was Fourier transformed and displayed. Each spectrum acquired for the reaction mixture was the assemble average over 200 measurements. 
A cylinder cell of 2.5-cm diameter and 5-cm length used as the reactor was settled in the compartment of the FTS. A volume of $15 \mathrm{~mL}$ of $0.1-0.14 \mathrm{M}(\mathrm{mol} / \mathrm{L})$ quadricyclane solution with metal salt, prepared in the range of $0.2-1.2 \mathrm{~g}$, was contained in the cell. When anhydrous $\mathrm{CuSO}_{4}$ (Merck) was used as the catalyst, quadricyclane solution was made in chloroform. When anhydrous $\mathrm{SnCl}_{2}$ (Janssen) was used, the solvent was changed to benzene. In either case, the solvent was kept still for $1 \mathrm{~h}$ to ensure that the solid may precipitate completely before acquiring the NIR spectra of the solvent. Then the appropriate amount of quadricyclane was added and kept still for $10 \mathrm{~min}$ before acquiring the NIR spectra of the reaction solution. The light beam was guided to propagate through the solution right above the solid surface. Since the suspended salts had settled down in the cell bottom, the effect of radiation scattering and sample opacity was minimized. The NIR spectrum was recorded at 5 -min intervals over a period of $6 \mathrm{~h}$.

Quadricyclane (quadricyclo[2.2.1.0.0] heptane) and norbornadiene (bicyclo[2.2.1] hepta-2,5-diene) (99\%, Aldrich) were used as purchased without further purification. The concentration of each compound was prepared in the range $0.005-0.11 \mathrm{M}$ for the partial least-squares analysis.

\section{Kinetic Modeling}

The behavior of reactant motion in the solid stacks results in different diffusion mechanisms. ${ }^{17}$ For the molecular mechanism, the pore size of the catalyst is larger than the mean free path, a distance that a molecule moves between collisions. Then the molecular motion behaves as in the solvent, neglecting disturbance by the catalyst. On the other hand, when the pore size is smaller than the mean free path, the wall collisions become important and the diffusion of the reactant is dominated by the Knudsen mechanism. ${ }^{17}$ The reactant diffusivity may depend on the diameter of the pore size and the mean molecular velocity.

For simplicity, we assume the diffusivity of quadricyclane inside the stacks of catalyst to be constant, $D$. Then the kinetic rate equation including the diffusion motion can be expressed as $^{18}$

$$
\frac{\mathrm{d} C}{\mathrm{~d} t}=D \frac{\mathrm{d}^{2} C}{\mathrm{~d} x^{2}}-k C^{n}
$$

where $C$ is the concentration of quadricyclane, $x$ is the depth of the stacks of catalyst, $n$ is the reaction order, and $k$ is the $n$-order rate constant for isomerization. In this work, the isomer conversion belongs to the first-order reaction, $n=1$. We assume that the catalyst surface may be covered steadily by the reactant during the reaction. Therefore, the steady-state condition may be applied, and eq 1 becomes

$$
D \frac{\mathrm{d}^{2} C}{\mathrm{~d} x^{2}}=k C
$$

The concentration in eq 2 becomes dimensionless by substitution of $C_{\mathrm{d}}=C / C_{\mathrm{s}}$, in which $C_{\mathrm{s}}$ indicates the steady-state concentration of quadricyclane covering the catalyst surface. The expression yields

$$
\frac{\mathrm{d}^{2} C_{\mathrm{d}}}{\mathrm{d} X^{2}}=C_{\mathrm{d}}
$$

where

$$
X=x \sqrt{k / D}
$$

One finds that $C=C_{\mathrm{s}}$ when quadricyclane is on the catalyst surface (i.e., $x \rightarrow 0$ ), while $C$ becomes zero when quadricyclane is far from the catalyst surface. Thus the boundary conditions are expressed as

$$
C_{\mathrm{d}}=1, \quad \text { as } X=0
$$

and

$$
C_{\mathrm{d}}=0 \text { and } \frac{\mathrm{d} C_{\mathrm{d}}}{\mathrm{d} X}=0, \quad \text { as } X=\infty
$$

Considering the boundary conditions, the solution to eq 3 yields

$$
C_{\mathrm{d}}=e^{-X}
$$

Substitution into the second derivative of $C$ with respect to $x$ gives rise to

$$
D \frac{\mathrm{d}^{2} C}{\mathrm{~d} x^{2}}=D C_{\mathrm{s}} \frac{\mathrm{d}^{2} C_{\mathrm{d}}}{\mathrm{d} x^{2}}=C_{\mathrm{s}} k e^{-x \sqrt{ } k / D}
$$

Equation $1(n=1)$ can be solved exactly with substitution of eq 8. Given the initial concentration, $C=C_{\mathrm{o}}$ at $t=0$, the solution yields

$$
C=C_{\mathrm{o}} e^{-k t}+\frac{P_{2}}{k}\left(1-e^{-k t}\right)
$$

where

$$
P_{2}=C_{\mathrm{s}} k e^{-x \sqrt{ } k / D}
$$

Equations 9 and 10 describe the concentration change of quadricyclane in the reaction evolution. Given the measured data of quadricyclane concentration, the reaction rate constant may be determined accordingly.

\section{Results and Discussion}

A. Determination of Isomer Concentrations. The chloroform solvent absorbs NIR radiation from 8000 to $11500 \mathrm{~cm}^{-1}$. When the background spectra is subtracted, only the major band at $8700 \mathrm{~cm}^{-1}$ leaves some noise in the baseline, as shown in Figure 1a. Fortunately, the NIR spectra of quadricyclane and norbornadiene in chloroform peak at 8840 and $8900 \mathrm{~cm}^{-1}$, respectively, such that the solvent background may lead to the least disturbance. Figure 1b shows the NIR spectra for conversion of quadricyclane to norbornadiene catalyzed by $\mathrm{CuSO}_{4}$ at reaction times of 10, 120, and $360 \mathrm{~min}$, respectively. The slight difference in the absorption bands of these two isomers allows us to use partial least squares regression to model and determine the individual concentration. ${ }^{19-21}$

When $\mathrm{SnCl}_{2}$ is used as the catalyst, the chloroform is changed to benzene. By analogy with the background spectrum subtraction described previously, we measure the NIR spectra of benzene and subtract from the reaction mixture. However, even after the background subtraction, the quadricyclane and norbornadiene are still buried in the large noise in the 8540-8850 $\mathrm{cm}^{-1}$ region. The NIR spectra for quadricyclane and norbornadiene can only be acquired in the range of $8850-9050 \mathrm{~cm}^{-1}$, in which their absorption intensities are stronger than the background spectra, as shown in Figure 2. The NIR spectra exhibit different features between quadricyclane and norbornadiene. As shown in Figure 2b, NIR spectra of the reaction mixture are recorded at the reaction times of 10, 60, 120, 240, and $360 \mathrm{~min}$, respectively. As the heterogeneous reaction 

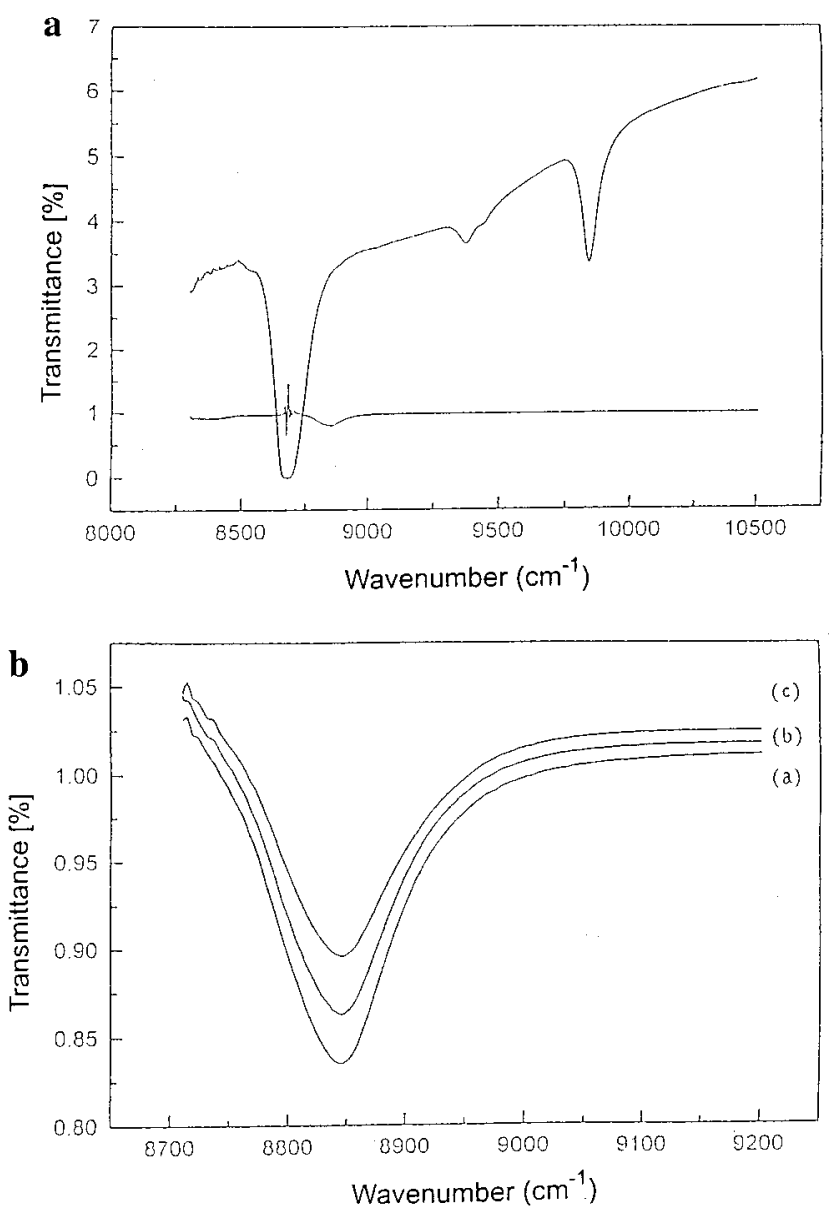

Figure 1. (a) Upper trace: the NIR absorption spectrum of chloroform lower trace: the NIR spectrum of quandricyclane in chloroform with the solvent background subtracted. A significant noise appears at 8700 $\mathrm{cm}^{-1}$ after the background subtraction. (b) The NIR spectra for isomerization of quadricyclane to norbornadiene catalyzed by $\mathrm{CuSO}_{4}$ over a reaction period of (a) $10 \mathrm{~min}$, (b) $120 \mathrm{~min}$, and (c) $360 \mathrm{~min}$, respectively.

evolves, the maximum absorption shifts to the position at 8900 $\mathrm{cm}^{-1}$, which indicates that the reaction mixture is predominated by the product.

In this work, more than 70 set of the NIR absorption spectra for each catalytic reaction system was acquired. With the analysis of partial least squares, either quadricyclane or norbornadiene concentration in the reaction mixture could be determined by a fit of the calibration set to the observed spectra. ${ }^{19-21}$ For the $\mathrm{CuSO}_{4}$ catalyst, 84 calibration set of various concentration combinations in the range $0.005-0.105 \mathrm{M}$ of quadricyclane and norbornadiene were made, while for the $\mathrm{SnCl}_{2}$ catalyst, 60 sets of concentration combinations in the same range were made. As shown in Figure $3 a$ and $3 b$, the fit between the predicted and actual concentrations for samples are characterized by a straight line with a regression coefficient $>0.98$, thereby ensuring reliability of the determined concentrations.

B. Determination of Rate Constants. Given the determined quadricyclane concentrations in the isomerization evolution, the fit of eq 9 gives rise to the pseudo-first-order rate constant, $k$, and $P_{2}$; the latter parameter is associated with the diffusion contribution. The weight of $\mathrm{CuSO}_{4}$ is varied from 0.2 to $1.2 \mathrm{~g}$. For each weight, we may obtain fitted parameters of $k$ and $P_{2}$, as listed in Table 1. Figure 4a gives an example for the decay of the quadricyclane concentration catalyzed by $\mathrm{CuSO}_{4}$ and the corresponding fit. The rate constants on the Table 1 appear to increase with the catalyst weight. The plot of $k$ as a function of
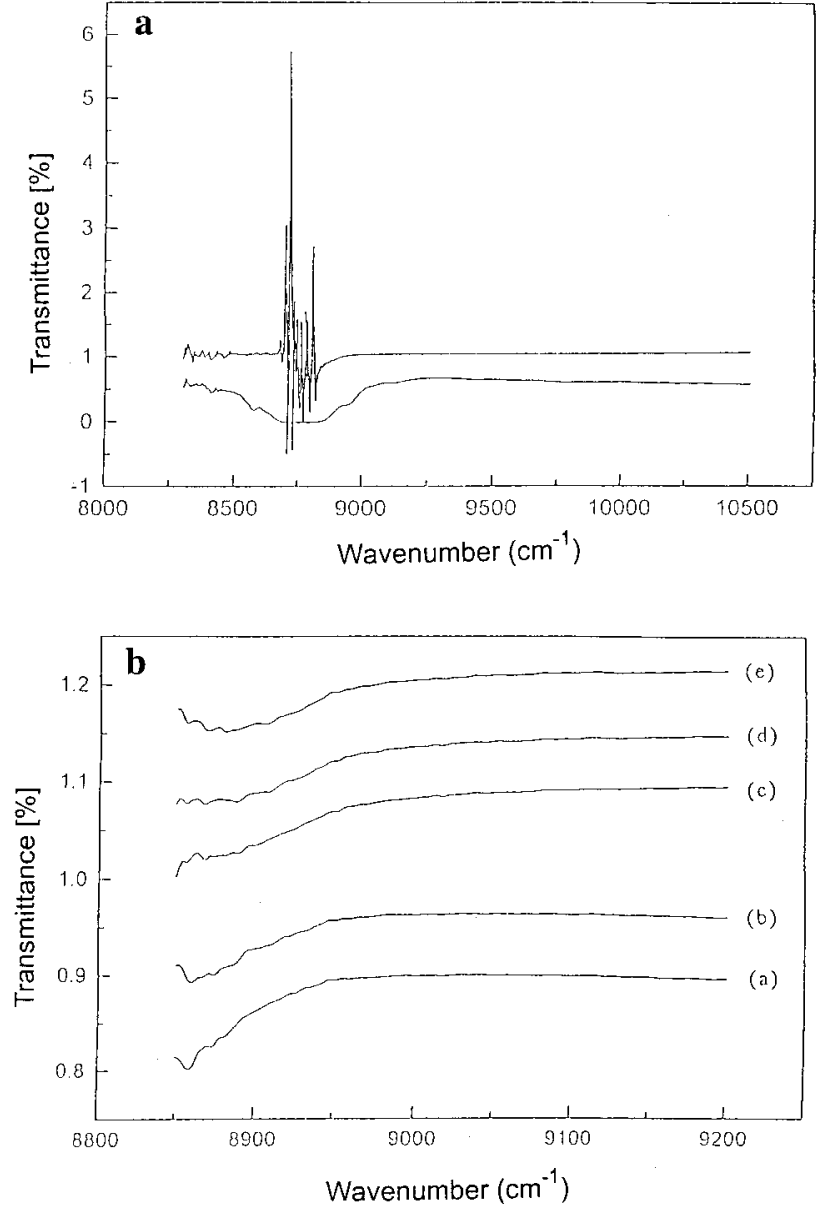

Figure 2. (a) Lower trace: the NIR spectrum of benzene; upper trace: the NIR spectrum of quadricyclane in benzene with the solvent background subtracted. A large portion of quadricyclane spectrum is buried in the noise even after background subtraction. (b) The NIR spectra for isomerization of quadricyclane to norbornadiene catalyzed by $\mathrm{SnCl}_{2}$ in benzene over a reaction period of (a) $10 \mathrm{~min}$, (b) $60 \mathrm{~min}$, (c) $120 \mathrm{~min}$, (d) $240 \mathrm{~min}$, and (e) $360 \mathrm{~min}$, respectively.

catalyst weight shows a straight line in Figure 4b, with a slope yielding the second-order rate constant, $k^{\mathrm{II}}=0.00138 \pm 0.00004$ $\min ^{-1} \mathrm{~g}^{-1}$.

Analogously, when $\mathrm{SnCl}_{2}$ is used as the catalyst in benzene, the corresponding rate constant and parameter $P_{2}$ are fitted from the decay of quadricyclane. The $\mathrm{SnCl}_{2}$ weight is varied from 0.2 to $1.2 \mathrm{~g}$. The obtained parameters for each weight of catalyst are listed in Table 2. The fit of the decay curve of quadricyclane concentration is also demonstrated in Figure 5a. The dependence of the pseudo-first-order rate constant on the $\mathrm{SnCl}_{2}$ weight is plotted in Figure 5b, yielding a straight line from which the second-order rate constant is determined to be $k^{\mathrm{II}}=0.00462 \pm$ $0.00009 \mathrm{~min}^{-1} \mathrm{~g}^{-1}$.

As the depth of catalyst stacks, $x$, in eq 10 is let to be zero, the steady-state concentration, $C_{\mathrm{s}}$, on the catalyst surface may be determined from the parameter, $P_{2}$. The results are also listed in Tables 1 and 2 for both catalysts. The $C_{\mathrm{s}}$ values seem to increase with the weight of catalyst and the initial concentration of quadricyclane, if we ignore the data with the smaller weights, which lead to a large relative error in the $P_{2}$ determination. However, the detailed relation between them is uncertain.

C. Comparison with Other Experiments. In the early work, Moore and co-workers have proposed that in the heterogeneous catalysis, the absorption of quadricyclane on the surface by combination of a one-site and a two-site coordination may lead to the production of norbornadiene. ${ }^{6}$ Therefore, the depletion 

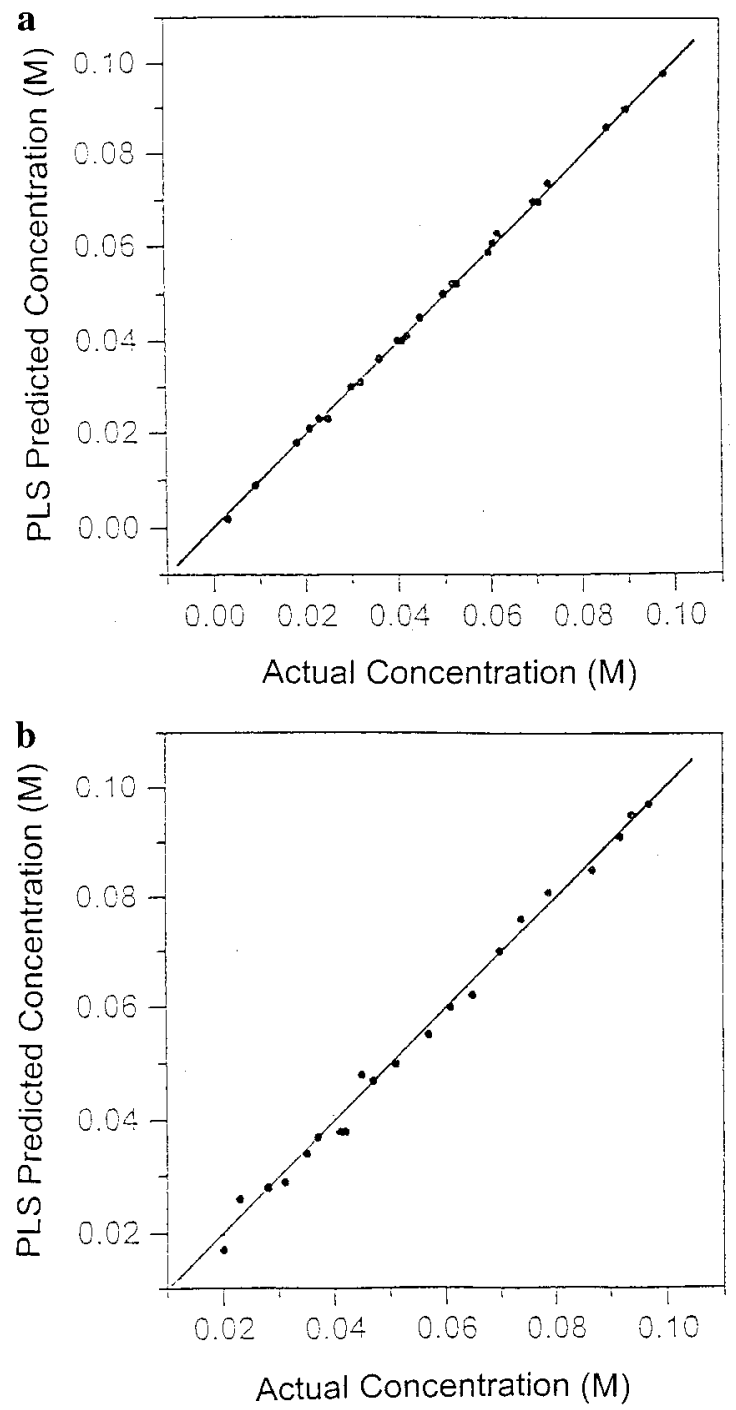

Figure 3. (a) Calibration plot for the determination of quadricyclane concentration from the NIR spectra of reaction mixture catalyzed by $\mathrm{CuSO}_{4}$ in chloroform. (b) Calibration plot for the determination of quadricyclane concentration from the NIR spectra of reaction mixture catalyzed by $\mathrm{SnCl}_{2}$ in benzene.

TABLE 1: The Kinetic Data of Quadricyclane Catalyzed by $\mathrm{CuSO}_{4}$

\begin{tabular}{ccccc}
\hline $\mathrm{CuSO}_{4}(\mathrm{~g})$ & $k\left(\times 10^{3} \mathrm{~min}^{-1}\right)$ & $P_{2}\left(\times 10^{5} \mathrm{M} \mathrm{min}^{-1}\right)$ & $\mathrm{Co}(\mathrm{M})^{a}$ & $\mathrm{Cs}(\mathrm{M})^{b}$ \\
\hline 1.2 & $1.57 \pm 0.13$ & $7 \pm 1$ & 0.1348 & 0.0446 \\
1.0 & $1.41 \pm 0.15$ & $6.0 \pm 1.6$ & 0.132 & 0.0426 \\
0.8 & $1.10 \pm 0.15$ & $3.0 \pm 1.5$ & 0.121 & 0.0273 \\
0.6 & $0.89 \pm 0.15$ & $1.0 \pm 1.6$ & 0.126 & 0.0112 \\
0.4 & $0.61 \pm 0.16$ & $1.0 \pm 1.7$ & 0.121 & 0.0164 \\
0.2 & $0.41 \pm 0.16$ & $1.0 \pm 1.7$ & 0.122 & 0.0244
\end{tabular}

${ }^{a}$ Initial concentration of quadricyclane. ${ }^{b}$ Steady-state concentration of quadricyclane on the catalyst surface.

rate of quadricyclane can be expressed as

$$
\frac{\mathrm{d} Q}{\mathrm{~d} t}=\left[k_{1}{ }^{\mathrm{II}} W+k_{2}{ }^{\mathrm{II}} W^{2}\right] Q
$$

where $Q$ denotes the quadricyclane concentration, and $W$ the weight of catalyst. They have determined the one-site and twosite coordinated rate constants, $k_{1}{ }^{\mathrm{II}}$ and $k_{2}{ }^{\mathrm{II}}$, to be $2.9 \times 10^{-2}$ $\min ^{-1} \mathrm{~g}^{-1}$ and $7.3 \times 10^{-2} \mathrm{~min}^{-1} \mathrm{~g}^{-2}$, respectively, for the $\mathrm{CuSO}_{4}$ catalyst in benzene by the detection of GC-MS. ${ }^{6}$

Vickers and co-workers have measured the kinetic behavior of quadricyclane and norbornadiene simultaneously by using
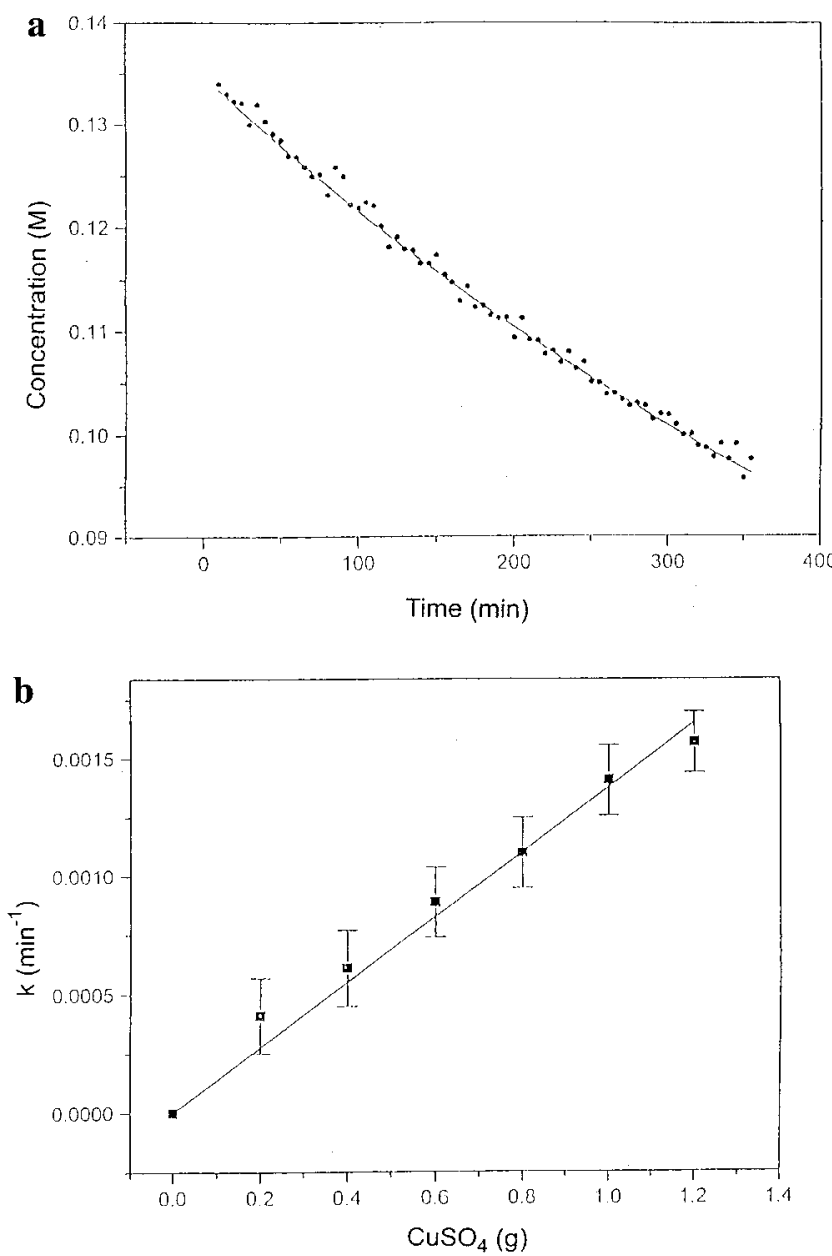

Figure 4. (a) Reaction time dependence of quadricyclane concentration catalyzed by $1.2 \mathrm{~g}$ of $\mathrm{CuSO}_{4}$ in chloroform. (b) Pseudo-first-order rate constant as a function of the weight of $\mathrm{CuSO}_{4}$. The second-order rate constant determined from the slope is $(1.38 \pm 0.04) \times 10^{-3} \mathrm{~min}^{-1}$ $\mathrm{g}^{-1}$.

TABLE 2: The Kinetic Data of Quadricyclane Catalyzed by $\mathrm{SnCl}_{2}$

\begin{tabular}{ccccc}
\hline $\mathrm{SnCl}_{2}(\mathrm{~g})$ & $k\left(\times 10^{3} \mathrm{~min}^{-1}\right)$ & $P_{2}\left(\times 10^{4} \mathrm{M} \mathrm{min}^{-1}\right)$ & $\mathrm{Co}(\mathrm{M})^{a}$ & $\mathrm{Cs}(\mathrm{M})^{b}$ \\
\hline 1.2 & $5.54 \pm 0.47$ & $2.3 \pm 0.3$ & 0.09 & 0.0415 \\
1.0 & $4.5 \pm 0.4$ & $2 \pm 0.3$ & 0.1 & 0.0444 \\
0.8 & $3.93 \pm 0.47$ & $1.5 \pm 0.3$ & 0.087 & 0.0382 \\
0.6 & $2.8 \pm 0.3$ & $0.8 \pm 0.2$ & 0.085 & 0.0286 \\
0.4 & $1.6 \pm 0.7$ & $0.5 \pm 0.6$ & 0.097 & 0.0313 \\
0.2 & $1.1 \pm 0.5$ & $0.2 \pm 0.4$ & 0.081 & 0.0182
\end{tabular}

${ }^{a}$ Initial concentration of quadricyclane. ${ }^{b}$ Steady-state concentration of quadricyclane on the catalyst surface.

Raman spectroscopy. ${ }^{7}$ They demonstrated that the temporal evolution of quadricyclane prepared initially at $0.0997 \mathrm{M}$ decreased almost exponentially to $0.03 \mathrm{M}$ within $240 \mathrm{~min}$, as catalyzed by $0.375 \mathrm{~g} \mathrm{CuSO}_{4}$ in chloroform. Given the above experimental conditions and the rate constants, $k_{1}{ }^{\mathrm{II}}$ and $k_{2}{ }^{\mathrm{II}}$, by Moore and co-workers, eq 11 yields the quadricyclane concentration to be $0.00058 \mathrm{M}$. The product concentration is about 2 orders of magnitude smaller than that obtained by Vickers and co-workers. ${ }^{7}$ The rate constants reported by Moore and coworkers are apparently much larger.

Given the rate constant, $k^{I I}=0.00138 \mathrm{~min}^{-1} \mathrm{~g}^{-1}$, for the $\mathrm{CuSO}_{4}$ catalyst in this work, eq 9 with substitution of the experimental conditions provided by Vickers and co-workers gives rise to a quadricyclane concentration to be $0.090 \mathrm{M}$. This value has the same order of magnitude as that by the Raman spectroscopy. The rate constant determined here seems to be 

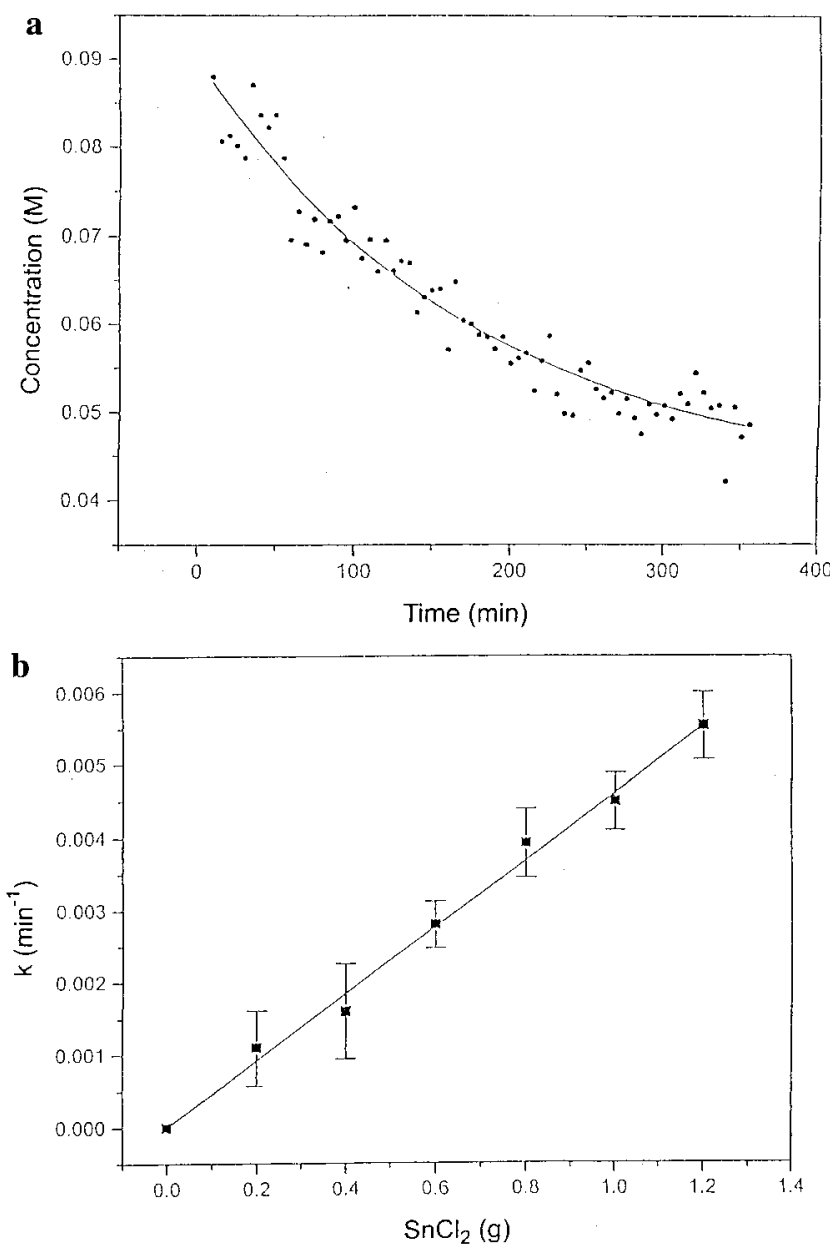

Figure 5. (a) Reaction time dependence of quadricyclane concentration catalyzed by $1.2 \mathrm{~g}$ of $\mathrm{SnCl}_{2}$ in benzene. (b) Pseudo-first-order rate constant as a function of the weight of $\mathrm{SnCl}_{2}$. The second-order rate constant determined from the slope is $(4.62 \pm 0.09) \times 10^{-3} \mathrm{~min}^{-1}$ $\mathrm{g}^{-1}$.

more reasonable than that by Moore and co-workers. ${ }^{6}$ It should be noted that the latter group used benzene as the solvent. It is uncertain whether the solvent effect may cause such great difference in the isomerization rate.

A distinct kinetic behavior for the heterogeneous catalysis under either a still or a stirred condition is reflected in three aspects. First, the depletion rates of quadricyclane in this work are expected to be slower than the system in which the solution is stirred to enhance the collision frequency of the reactants. That consideration interprets why the estimated quadricyclane concentration based on our rate constant is about three times larger than that obtained by Vickers and co-workers. Second, the measured decay of quadricyclane concentration in this work cannot be well fitted by using eq 11 derived by Moore and coworkers. Since our reaction system is kept still without stirring, the formation of a two-site coordination (1:2 complex) between reactant and catalyst should be negligible. That is, $k_{2}{ }^{\mathrm{II}}$ is ignored, and $k_{1}{ }^{\mathrm{II}}$ is equivalent to our obtained $k^{I I}$. Third, the isomerization rate should take into account the diffusion contribution as in this work, which is ignored in the kinetics for a continuously stirred heterogeneous solution.

The isomerization rate catalyzed by $\mathrm{SnCl}_{2}$ in benzene is found to be three times faster than that by $\mathrm{CuSO}_{4}$ in chloroform. However, to our knowledge, we cannot find appropriate heterogeneous reactions reported for comparison. Some rate constants have been measured under different conditions. ${ }^{8,13}$ For instance, Patrick and co-workers have obtained a rate constant of $2 \times 10^{-7}(\mathrm{~mol} \mathrm{Sn} / \mathrm{L})^{-1} \mathrm{~s}^{-1}$ with an effective polymeranchored $\mathrm{SnCl}_{2}$ catalyst. ${ }^{13}$ By using NMR spectroscopy, the same group later obtained a rate constant of $1.82 \times 10^{-4} \mathrm{M}^{-1}$ $\mathrm{s}^{-1}$ by dissolving $50 \mathrm{mg}$ of $\mathrm{SnCl}_{2}$ in $1 \mathrm{~mL}$ of $\mathrm{CD}_{3} \mathrm{OD} .^{8}$

\section{Conclusion}

By using Fourier transform NIR absorption spectroscopy, we have determined the kinetic data for the catalytic reaction of quadricyclane. For a heterogeneous reaction, the suspended solid may increase the sample opacity and cause the problem of radiation scattering. In this work, the reaction mixture is kept still to prevent from the effect of sample heterogeneity. The NIR radiation is guided to pass through the solution above the solid surface. Accordingly, a kinetic model taking into account the contribution of diffusion motion has been derived to give rise to the related kinetic parameters. The obtained depletion rate of quadricyclane is linearly proportional to the weight of catalyst. That is, the norbornadiene is produced via a one-site coordination (1:1 complex) between the reactant and the catalyst. The product contribution from the intermediate of a two-site coordination becomes negligible in our system. Despite a distinct kinetic mechanism resulting from a still reaction medium, the obtained rate constants are reliable. The depletion rates of quadricyclane are found to be on the same order of magnitude as that by using Raman spectroscopy in a continuously stirred solution. Therefore, in this work we have successfully provided an alternative technique for the kinetic measurement of heterogeneous catalysis

Acknowledgment. This work is supported by the National Science Council and Chinese Petroleum Company of the Republic of China under Contract No. NSC89-2119-M-002007. We thank C. B. Ke for preparing the plots.

\section{References and Notes}

(1) Hautala, R. R.; King, R. B.; Kutal, C. Solar Energy: Chemical Conversion and Storage; Humana Press: Clifton, NJ, 1979; p 333-344.

(2) Bren, V. A.; Dubonosov, A. D.; Minkin, V. I.; Chernoivanov, V. A. Russ. Chem. Rev. 1991, 60, 451.

(3) Cuppoletti, A.; Dinnocenzo, J. P.; Goodman, J. L.; Gould, I. R. J. Phys. Chem. A 1999, 103, 11253.

(4) Sluggett, G. W.; Turro, N. J.; Roth, H. D. J. Phys. Chem. A 1997, $101,8834$.

(5) Franceschi, F.; Guardigli, M.; Solari, E.; Floriani, C.; Chiesi, V. A.; Rizzoli, C. Inorg. Chem. 1997, 36, 4099.

(6) Fife, D. J.; Morse, K. W.; Moore, W. M. J. Am. Chem. Soc. 1983 , 105, 7404. 592.

(7) Ford, J. F.; Mann, C. K.; Vickers, T. J. Appl. Spectrosc. 1994, 48,

(8) Patrick, T. B.; Bechtold, D. S. J. Org. Chem. 1984, 49, 1935.

(9) Noyori, R.; Umeda, I.; Kawauchi, H.; Takaya, H. J. Am. Chem. Soc. 1975, 97, 812 .

(10) Manassen, J. J. Catal. 1970, 18, 38

(11) Taylor, R. B.; Jennings, P. W. Inorg. Chem. 1981, 20, 3997.

(12) Sen, A.; Thomas, R. R. Organometallics 1982, 1, 1251.

(13) Landis, M. E.; Gremaud, D.; Patrick, T. B. Tetrahedron Lett. 1982, 23,375

(14) Wang, C. C.; Chin, T. L.; Lin, K. C. J. Chem. Phys. 1997, 107, 10348 .

(15) Chin, T. L.; Lin, K. C. Appl. Spectrosc. 1999, 53, 22

(16) Wang, C. C.; Chen, Y. P.; Chin, T. L.; Lin, K. C. J. Chem. Phys. 2000, 112, 10204

(17) Karger, J.; Ruthven, D. M. Diffusion in Zeolities and Other Microporous Solids; Wiley Press: New York, 1992.

(18) Crank, J. The Mathmatics of Diffusion; Oxford University Press: Ely House, London, 1975.

(19) Lindberg, W.; Persson, J.-A. Anal. Chem. 1983, 55, 643.

(20) Phelan, M. K.; Barlow, C. H.; Kelly, J. J.; Jinguji, T. M.; Callis, J. B. Anal. Chem. 1989, 61, 1419.

(21) Schrieve, G. D.; Ullman, A. H. Appl. Spectrosc. 1991, 45, 713. 